\title{
Magnon-magnon entanglement and its quantification via a microwave cavity
}

\author{
Vahid Azimi Mousolou, ${ }^{1,2, *}$ Yuefei Liu, ${ }^{3}$ Anders Bergman, ${ }^{2}$ Anna Delin, ${ }^{2,3,4}$ Olle Eriksson, ${ }^{2,5}$ \\ Manuel Pereiro, ${ }^{2}$ Danny Thonig, ${ }^{5}$ and Erik Sjöqvist ${ }^{2, \dagger}$ \\ ${ }^{1}$ Department of Applied Mathematics and Computer Science, Faculty of Mathematics and Statistics, \\ University of Isfahan, Isfahan 81746-73441, Iran \\ ${ }^{2}$ Department of Physics and Astronomy, Uppsala University, Box 516, SE-751 20 Uppsala, Sweden \\ ${ }^{3}$ Department of Applied Physics, School of Engineering Sciences, KTH Royal Institute of Technology, \\ AlbaNova University Center, SE-10691 Stockholm, Sweden \\ ${ }^{4}$ Swedish e-Science Research Center, KTH Royal Institute of Technology, SE-10044 Stockholm, Sweden \\ ${ }^{5}$ School of Science and Technology, Örebro University, SE-701 82 Örebro, Sweden
}

(Received 10 June 2021; revised 16 November 2021; accepted 22 November 2021; published 8 December 2021)

\begin{abstract}
Quantum magnonics is an emerging research field, with great potential for applications in magnon based hybrid systems and quantum information processing. Quantum correlation, such as entanglement, is a central resource in many quantum information protocols that naturally comes about in any study toward quantum technologies. This applies also to quantum magnonics. Here, we investigate antiferromagnetic coupling of two ferromagnetic sublattices that can have two different magnon modes. We show how this may lead to experimentally measurable bipartite continuous-variable magnon-magnon entanglement. The entanglement can be fully characterized via a single squeezing parameter or, equivalently, entanglement parameter. The clear relation between the entanglement parameter and the Einstein, Podolsky, and Rosen (EPR) function of the ground state opens up for experimental quantification magnon-magnon continuous-variable entanglement and EPR nonlocality. We propose a practical experimental realization to measure the EPR function of the ground state, in a setting that relies on magnon-photon interaction in a microwave cavity.
\end{abstract}

DOI: 10.1103/PhysRevB.104.224302

\section{INTRODUCTION}

Hybrid quantum systems provide a natural flexible platform for quantum technologies. Recent developments in quantum magnon spintronics suggest that hybrid quantum systems based on collective spin-wave excitations in magnetic materials, i.e., magnons, are highly promising for many short and long term applications in quantum technologies, including quantum sensing, quantum communication, quantum simulation, and quantum computing [1-4]. The spin-wave magnon modes interact coherently with microwave and optical photons, phonons, and superconducting qubits, which is essential for engineering efficient hybrid quantum technologies [3,4].

Recently, studies of entanglement between different components of a hybrid magnonic system have been under focus, as a fundamental element in a quantum device design [3-11]. Relevant for the present paper, it was suggested in Ref. [11] that one may identify a hierarchy of magnon-mode entanglement in antiferromagnetic materials. The motivation for studies of entanglement in magnetic materials can be traced

\footnotetext{
*v.azimi@sci.ui.ac.ir

†erik.sjoqvist@physics.uu.se
}

Published by the American Physical Society under the terms of the Creative Commons Attribution 4.0 International license. Further distribution of this work must maintain attribution to the author(s) and the published article's title, journal citation, and DOI. Funded by Bibsam. to the development of modern synthetic routes that allow most combinations of elements to form a magnetic material with tailored properties. This development in materials growth, in combination with advanced lithographic techniques for nanostructuring, open up new vistas to be explored in magnetic nanotechnology [12], where magnon-magnon entanglement is an essentially unexplored research field.

Here, we discuss how an antiferromagnetic coupling between two ferromagnetic spin lattices creates bipartite continuous-variable entanglement between the two ferromagnetic magnon modes in a way that each energy eigenstate of the system becomes a two-mode coherent state with nonzero entropy of entanglement. We show that the entanglement entropy of the energy eigenstates is given by a single squeezing parameter, which can be related and measured through the Einstein, Podolsky, and Rosen (EPR) function of the ground state of the system. We also propose a feasible setup for experimental measurement of the EPR function and consequently the degree of bipartite ferromagnetic magnon-mode entanglement and EPR nonlocality. The measurement setup is based on magnon-photon coupling in a microwave cavity, which is appropriate for a wide range of antiferromagnetic materials [13] or synthetic antiferromagnetic multilayers [14]. There are many compounds with antiferromagnetism, where oxides comprise a broad class. In the perovskite structure alone, there are three types of antiferromagnetic structures that are relevant for the discussion presented here; A-, C-, and G-type antiferromagnetism $[15,16]$. Synthetically grown multilayers that have ferromagnetic coupling within one layer 


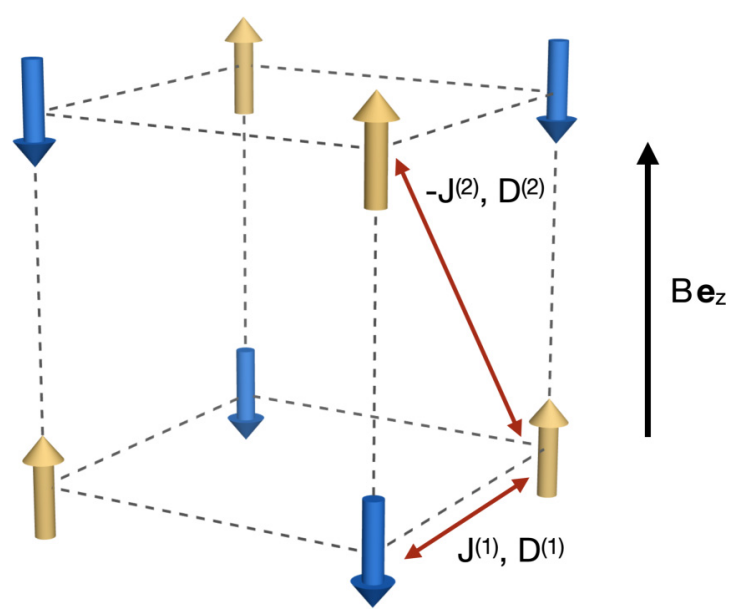

FIG. 1. Schematic illustration of a bipartite system consisting of two ferromagnetic spin sublattices with opposite magnetizations, which are coupled antiferromagnetically to each other. The whole system can be viewed as a single antiferromagnetic compound with nearest- and next-nearest-neighbor interactions given by Heisenberg and Dzyaloshinskii-Moriya exchange interactions. The superfix (1) denotes couplings between the two different sublattices while (2) describing couplings within the same sublattice.

and antiferromagnetic coupling between the layers are also plentiful in the literature, and form in fact the basis for the giant magnetoresistance effect [17].

\section{ESSENTIAL ASPECTS OF MAGNON-MAGNON ENTANGLEMENT}

In this section we describe briefly the Hamiltonian considered in this paper, and the main conclusion with regard to which magnon modes are most suitable for quantification of magnon-magnon entanglement in an experimental setup. It should be noted that this section has some overlap with the analysis presented in Ref. [11], where the most central findings are included in order to make the present presentation self-contained. A full account of the technical aspects of this discussion can be found in the Appendices. We consider a bipartite system of two ferromagnetic sublattices with opposite magnetizations denoted here by $A$ and $B$, which are coupled antiferromagnetically to each other. Figure 1 illustrates the considered spin model system for a G-type antiferromagnet structure, which is consistent with the formulation for the Hamiltonian in Eq. (2). However, the following discussion is general and holds for every bipartite antiferromagnetic structure including $\mathrm{C}$ and $\mathrm{A}$ types, where the latter also allows for describing synthetically grown antiferromagnets.

The magnetic interactions of the G-type system are described by the Hamiltonian

$$
H=H_{1}+H_{2}+H_{a}+H_{z},
$$

where

$$
\begin{aligned}
& H_{1}=\sum_{\langle i j\rangle}\left[J^{(1)} \mathbf{S}_{i} \cdot \mathbf{S}_{j}+\mathbf{D}_{i j}^{(1)} \cdot\left(\mathbf{S}_{i} \times \mathbf{S}_{j}\right)\right], \\
& H_{2}=\sum_{\langle\langle i j\rangle\rangle}\left[-J^{(2)} \mathbf{S}_{i} \cdot \mathbf{S}_{j}+\mathbf{D}_{i j}^{(2)} \cdot\left(\mathbf{S}_{i} \times \mathbf{S}_{j}\right)\right],
\end{aligned}
$$

$$
\begin{aligned}
& H_{a}=-\mathcal{K} \sum_{i}\left(S_{i}^{z}\right)^{2}, \quad \mathcal{K}>0, \\
& H_{z}=\sum_{i} \mathbf{B} \cdot \mathbf{S}_{i}, \quad \mathbf{B}=B \mathbf{e}_{z}, \\
& J^{(1)}>0, \quad \mathbf{D}_{i j}^{(1)}=-\mathbf{D}_{j i}^{(1)}=\mathbf{D}^{(1)}=D^{(1)} \mathbf{e}_{z}, \\
& J^{(2)}>0, \quad \mathbf{D}_{i j}^{(2)}=-\mathbf{D}_{j i}^{(2)}=\mathbf{D}^{(2)}=D^{(2)} \mathbf{e}_{z} .
\end{aligned}
$$

$H_{1}$ defines antiferromagnetic interaction between neighboring sites on opposite sublattices, e.g., nearest-neighbor spins in Gtype antiferromagnets; $H_{2}$ defines ferromagnetic interaction between neighboring sites within each sublattices, e.g., nextnearest-neighbor spins in G-type antiferromagnets. In our model, we assume that the interaction can be described by using a Heisenberg (symmetric) exchange term combined with a Dzyaloshinskii-Moriya (DM) (antisymmetric) exchange term. $H_{a}$ is the easy axis anisotropy, and $H_{z}$ represents the Zeeman term in the Hamiltonian. Both $H_{a}$ and $H_{z}$ are taken to be in the $z$ direction for each spin regardless of sublattice.

At low temperatures $\left(k_{B} T \ll \min \left\{J^{(1)}, J^{(2)}\right\}\right)$, one may bosonize the Hamiltonian in terms of collective modes in $\mathbf{k}$ space to arrive at

$$
\begin{aligned}
H_{\mathbf{k}}= & \omega_{a_{\mathbf{k}}} a_{\mathbf{k}}^{\dagger} a_{\mathbf{k}}+\omega_{b_{-\mathbf{k}}} b_{-\mathbf{k}}^{\dagger} b_{-\mathbf{k}} \\
& +g_{\mathbf{k}} a_{\mathbf{k}} b_{-\mathbf{k}}+g_{\mathbf{k}}^{*} a_{\mathbf{k}}^{\dagger} b_{-\mathbf{k}}^{\dagger} .
\end{aligned}
$$

Here,

$$
\begin{aligned}
\omega_{a_{\mathbf{k}}}= & \epsilon_{\mathbf{k}}-B, \quad \omega_{b_{-\mathbf{k}}}=\epsilon_{\mathbf{k}}+B, \\
\epsilon_{\mathbf{k}}= & S\left(z^{(1)} J^{(1)}+2 \mathcal{K}\right. \\
& \left.+z^{(2)}\left\{J^{(2)}-2 \operatorname{Re}\left[\left(J^{(2)}-i D^{(2)}\right) \gamma_{\mathbf{k}}^{(2)}\right]\right\}\right), \\
g_{\mathbf{k}}= & S z^{(1)} \gamma_{\mathbf{k}}^{(1)}\left(J^{(1)}+i D^{(1)}\right),
\end{aligned}
$$

and $a_{\mathbf{k}}^{\dagger}\left(a_{\mathbf{k}}\right)$ and $b_{-\mathbf{k}}^{\dagger}\left(b_{-\mathbf{k}}\right)$ are bosonic creation (annihilation) operators, which commute and define independent families of bosonic operators on the opposite sublattices $A$ and $B$, respectively.

As derived in Eq. (A8) of Appendix A, under SU(1,1) Bogoliubov transformation, it is possible to obtain the following diagonal form of the Hamiltonian:

$$
H_{\mathbf{k}}=\omega_{\alpha_{\mathbf{k}}} \alpha_{\mathbf{k}}^{\dagger} \alpha_{\mathbf{k}}+\omega_{\beta_{-\mathbf{k}}} \beta_{-\mathbf{k}}^{\dagger} \beta_{-\mathbf{k}},
$$

where the bosonic operators $\alpha$ and $\beta$ are linear combinations of the sublattice bosonic operators $a$ and $b$. From this the complete energy eigenbasis states of the form $\left(\alpha_{\mathbf{k}}^{\dagger}\right)^{x}\left(\beta_{-\mathbf{k}}^{\dagger}\right)^{y}\left|\psi_{0}\right\rangle=$ $\sqrt{x ! y !}\left|x ; \alpha_{\mathbf{k}}\right\rangle\left|y ; \beta_{-\mathbf{k}}\right\rangle$ for any positive integer powers of $x$ and $y$, where

$$
\left|\psi_{0}\left(r_{\mathbf{k}}, \phi_{\mathbf{k}}\right)\right\rangle=\frac{1}{\cosh r_{\mathbf{k}}} \sum_{n=0}^{\infty} e^{i n \phi_{\mathbf{k}}} \tanh ^{n} r_{\mathbf{k}}\left|n ; a_{\mathbf{k}}\right\rangle\left|n ; b_{-\mathbf{k}}\right\rangle
$$

describes the ground state $\left|\psi_{0}\right\rangle$ in the $(a, b)$ modes as a function of $\left(r_{\mathbf{k}}, \phi_{\mathbf{k}}\right)$ given by Eq. (A7), are explicitly specified in Appendix B. Here, we make use of the fact that entanglement entropies of all energy eigenbasis states in the $(a, b)$ modes can be written as

$$
E\left[\left(\alpha_{\mathbf{k}}^{\dagger}\right)^{x}\left(\beta_{-\mathbf{k}}^{\dagger}\right)^{y}\left|\psi_{0}\right\rangle\right]=-\sum_{n=0}^{\infty}\left|p_{n ; \mathbf{k}}^{(x, y)}\right|^{2} \log \left|p_{n ; \mathbf{k}}^{(x, y)}\right|^{2}
$$


where $\left|p_{n ; \mathbf{k}}^{(x, y)}\right|$ are only functions of the single parameter $r_{\mathbf{k}}$. Detailed analyses in Appendix B show that the entanglement parameter $r_{\mathbf{k}}$ and therefore the entanglement entropies are only given by the absolute value of the ratio $\Gamma_{\mathbf{k}} \propto$ $\frac{g_{\mathbf{k}}}{\omega_{a_{\mathbf{k}}}+\omega_{b_{-}}}=\frac{g_{\mathbf{k}}}{\epsilon_{\mathbf{k}}}$. As far as the ground state $\left|\psi_{0}\right\rangle$ is concerned the entanglement parameter $r_{\mathbf{k}}$ is the same as the squeezing parameter discussed in Ref. [18].

In the absence of an antiferromagnetic coupling term $H_{1}$ in the Hamiltonian in Eqs. (1), the $(a, b)$ and $(\alpha, \beta)$ modes coincide and as a result the bosonized Hamiltonians in Eqs. (3) and (5) would be exactly the same describing two separate noninteracting ferromagnets with opposite magnetizations, e.g., aligned in the $z$ direction so that $\left\langle\mathbf{S}_{i \in A}\right\rangle=-\left\langle\mathbf{S}_{j \in B}\right\rangle=$ $(0,0, S)$. In this case, the full energy spectra are separately given by the ferromagnetic bosonic modes of $a$ and $b$. Hence, $H_{1}=0$ corresponds to a pair of disentangled ferromagnets with separable two-magnon-mode eigenstates. As soon as the antiferromagnetic interaction between the two ferromagnetic sublattices is turned on, i.e., $H_{1} \neq 0$, the $(\alpha, \beta)$ modes become hybridized antiferromagnetic magnon modes while $(a, b)$ still represents a pair of ferromagnetic magnon modes quantizing the two ferromagnet sublattices. Indeed, finite entanglement entropies between $a$ and $b$ magnon modes in the case of $H_{1} \neq 0$ indicate that the antiferromagnetic interaction creates bipartite continuous-variable entanglement between the two ferromagnets in a way that each energy eigenstate becomes an entangled two-magnon-mode coherent state (see Appendix $\mathrm{B}$ as well as Ref. [11]). Each of the energy eigenstates is a coherent superposition of joint excitations of ferromagnetic magnons in $a$ and $b$ modes.

Based on the above considerations, a hierarchy of magnonmode entanglement in antiferromagnets of the kind discussed here has been analyzed in detail in Ref. [11]. The theoretical analysis in Ref. [11] was mainly focused on the ground state of the system, while we here extend the discussion to cover excited states as well. What follows is the main focus of the present paper: to provide a theoretical foundation, which establishes an experimental platform to measure entanglement between magnon modes of an antiferromagnetic material, as described by a pair of bosonic modes $(a, b)$, through the EPR type of nonlocality. The analytical work is combined with a suggested experimental setup, that relies on the magnon-photon interaction, with which realistic experimental investigations can be made. This hence provides a formal, as well as practical, platform for which magnon entanglement may be quantified experimentally.

\section{MEASUREMENT SCHEME VIA THE MICROWAVE CAVITY}

A highly relevant concept to continuous-variable entanglement is the Bell-type nonlocal correlations known as EPR nonlocality. In the present case, the EPR nonlocality can be quantified by the following EPR function [19]:

$$
\Delta(\psi)=\frac{1}{2}\left[\operatorname{Var}_{\psi}\left(X_{\mathbf{k}}^{A}+X_{\mathbf{k}}^{B}\right)+\operatorname{Var}_{\psi}\left(P_{\mathbf{k}}^{A}-P_{\mathbf{k}}^{B}\right)\right],
$$

where $X_{\mathbf{k}}^{A}=\frac{a_{\mathbf{k}}+a_{\mathbf{k}}^{\dagger}}{\sqrt{2}} \quad\left(X_{\mathbf{k}}^{B}=\frac{b_{\mathbf{k}}+b_{\mathbf{k}}^{\dagger}}{\sqrt{2}}\right)$ and $P_{\mathbf{k}}^{A}=\frac{a_{\mathbf{k}}-a_{\mathbf{k}}^{\dagger}}{i \sqrt{2}} \quad\left(P_{\mathbf{k}}^{B}=\right.$ $\left.\frac{b_{\mathbf{k}}-b_{\mathbf{k}}^{\dagger}}{i \sqrt{2}}\right)$ are assumed to be the dimensionless position and
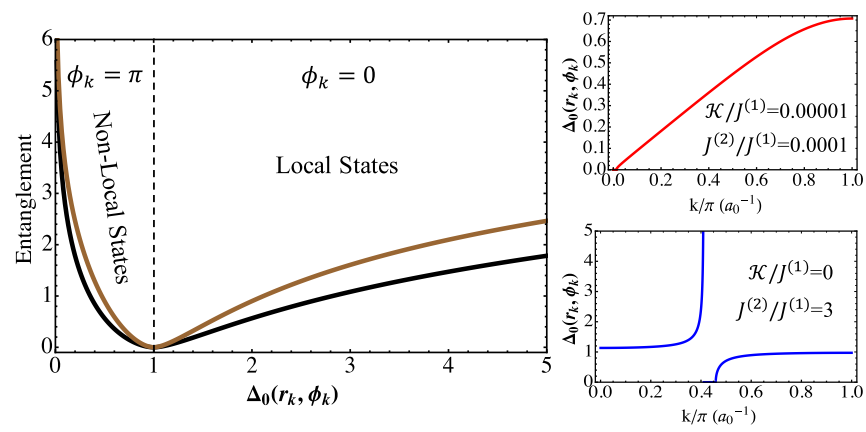

FIG. 2. Left panel: Entanglement entropies $E\left[\left|\psi_{0}\left(r_{\mathbf{k}}, \phi_{\mathbf{k}}\right)\right\rangle\right]$ and $E\left[\alpha_{\mathbf{k}}^{\dagger}\left|\psi_{0}\left(r_{\mathbf{k}}, \phi_{\mathbf{k}}\right)\right\rangle\right]=E\left[\beta_{-\mathbf{k}}^{\dagger}\left|\psi_{0}\left(r_{\mathbf{k}}, \phi_{\mathbf{k}}\right)\right\rangle\right]$ against the EPR function $\Delta_{0}\left(r_{\mathbf{k}}, \phi_{\mathbf{k}}\right)$ for antiferromagnetic spin lattices with only Heisenberg interactions. Black and brown curves correspond to the entanglement of the ground state and the first excited state, respectively. Right panels illustrate how the EPR function $\Delta_{0}\left(r_{\mathbf{k}}, \phi_{\mathbf{k}}\right)$ depends on $\mathbf{k}$ along the $(0,0,1)$ direction in a simple cubic lattice for selected values of exchange couplings $J^{(1)}$ and $J^{(2)}$, and magnetic anisotropy $\mathcal{K}$.

momentum quadratures for the $a_{\mathbf{k}}\left(b_{\mathbf{k}}\right)$ mode, respectively. $\operatorname{Var}_{\psi}(V)$ is the variance of a Hermitian operator $V$ with respect to the state $|\psi\rangle$. The uncertainty relation $\Delta(\psi) \geqslant 1$ is known to hold for any given bipartite separable state $|\psi\rangle$ [19]. Therefore, any violation of this inequality is an indication of the state $|\psi\rangle$ being nonlocal and indeed a bipartite entangled state. Note that the EPR nonlocality specifies a stronger type of entanglement than a nonzero entropy of entanglement in the sense that there are states with nonzero entropy of entanglement which do not violate the uncertainty relation. Note also that the EPR nonlocality depends, just like the entanglement entropy, on the modes that are chosen. In the present discussion we consider $(a, b)$ modes.

For the spin-wave ground state $\left|\psi_{0}\left(r_{\mathbf{k}}, \phi_{\mathbf{k}}\right)\right\rangle$ given in Eq. (B6), we obtain the EPR function

$$
\Delta_{0}\left(r_{\mathbf{k}}, \phi_{\mathbf{k}}\right)=\cosh 2 r_{\mathbf{k}}+\sinh 2 r_{\mathbf{k}} \cos \phi_{\mathbf{k}},
$$

which specifies the relation between the entanglement parameter and EPR nonlocality. Since the ground state EPR nonlocality and the entanglement entropies depend on the same squeezing parameter, one may analyze the dependence between entanglement entropy and the ground state EPR nonlocality. Figure 2 illustrates the two-mode magnon entanglement in the ground state and first excited states against the EPR function $\Delta_{0}\left(r_{\mathbf{k}}, \phi_{\mathbf{k}}\right)$, for antiferromagnetic spin lattices, where only Heisenberg interactions are relevant (DM interactions are neglected) and described by Eqs. (1) and (2). Typical materials that are known to be described by this type of spin Hamiltonian are antiferromagnets like $\mathrm{BiFeO}_{3}$ [20] and $\mathrm{LaMnO}_{3}$ [21], as well as synthetic, antiferromagnetic multilayers [17]. In this case, $\Gamma_{\mathbf{k}}$ is real valued and

$$
\Delta_{0}\left(r_{\mathbf{k}}, \phi_{\mathbf{k}}\right)=\left\{\begin{array}{lll}
e^{2 r_{\mathbf{k}}}, & \text { if } \quad \phi_{\mathbf{k}}=0\left(\Gamma_{\mathbf{k}}<0\right), \\
e^{-2 r_{\mathbf{k}}}, & \text { if } \quad \phi_{\mathbf{k}}=\pi\left(\Gamma_{\mathbf{k}}>0\right) .
\end{array}\right.
$$

Two distinct regions, the nonlocal bipartite entangled state and the local bipartite entangled state with transition point at $\Delta_{0}\left(r_{\mathbf{k}}, \phi_{\mathbf{k}}\right)=1$, in Fig. 2 distinguish the stronger region of magnon-magnon entanglement by the EPR uncertainty 
relation $\Delta_{0}\left(r_{\mathbf{k}}, \phi_{\mathbf{k}}\right)<1$. The clear relation between the EPR function and the two-mode magnon entanglement entropy allows for experimental quantification of magnon-magnon entanglement, as we discuss in detail below. It is worth mentioning that the EPR nonlocality has been used for verification of entanglement in optical and atomic systems based on homodyne detection and types of interferometry setups [22-28]. However, these types of measurement setups are not realistic for magnon systems, since these technologies are mainly based on beam splitters that have limitations for characterizing magnon entanglement. We propose as a solution a mechanism and measurement setup that relies on light-matter interaction as a probe to observe the EPR function and thus EPR nonlocality and the degree of magnon-magnon entanglement.

Cavity modes can couple to magnon modes in both antiferromagnets and ferromagnets [29-34]. The magnon-photon interaction can be understood in terms of the vector potential of the quantized photon field and the magnetic moments of the material. Since the mode polarization and propagation direction of the electromagnetic wave are highly relevant [33], we first introduce the essential physics of this interaction. After this, a possible structure of an experimental measurement of magnon entanglement is discussed.

We assume a microwave cavity electromagnetic field described by the vector potential

$$
\begin{aligned}
\mathbf{A}_{\mathbf{k}}(\mathbf{r}, t) & =\mathbf{A}_{L ; \mathbf{k}}(\mathbf{r}, t)+\mathbf{A}_{R ; \mathbf{k}}(\mathbf{r}, t), \\
\mathbf{A}_{R ; \mathbf{k}}(\mathbf{r}, t) & =A_{0}\left[\mathbf{e}_{R} c_{\mathbf{k}} e^{-i(\mathbf{k} \cdot \mathbf{r}+\omega t)}+\mathbf{e}_{R}^{*} c_{\mathbf{k}}^{\dagger} e^{i(\mathbf{k} \cdot \mathbf{r}+\omega t)}\right] \\
& =e^{i t \omega c_{\mathbf{k}}^{\dagger} c_{\mathbf{k}}} \mathbf{A}_{R ; \mathbf{k}}(\mathbf{r}, 0) e^{-i t \omega c_{\mathbf{k}}^{\dagger} c_{\mathbf{k}}}, \\
\mathbf{A}_{L ; \mathbf{k}}(\mathbf{r}, t) & =A_{0}\left[\mathbf{e}_{L} d_{-\mathbf{k}} e^{i(\mathbf{k} \cdot \mathbf{r}-\omega t)}+\mathbf{e}_{L}^{*} d_{-\mathbf{k}}^{\dagger} e^{-i(\mathbf{k} \cdot \mathbf{r}-\omega t)}\right] \\
& =e^{i t \omega d_{-\mathbf{k}}^{\dagger} d_{-\mathbf{k}}} \mathbf{A}_{L ; \mathbf{k}}(\mathbf{r}, 0) e^{-i t \omega d_{-\mathbf{k}}^{\dagger} d_{-\mathbf{k}}}
\end{aligned}
$$

for a given $\mathbf{k}$ vector describing the propagation direction of the electromagnetic wave. $A_{0}$ is the amplitude of the vector potential and $\omega$ is the single cavity mode frequency, which are both tuned by the volume of the cavity and the separation distance between the two conductor plates in the cavity (Fig. 2). Here, we focus on the lowest energy cavity mode and disregard contributions from the higher energy cavity modes. In fact, the vector potential represents superposition of right and left circularly polarized photons, where $c_{\mathbf{k}}\left(c_{\mathbf{k}}^{\dagger}\right)$ and $d_{-\mathbf{k}}\left(d_{-\mathbf{k}}^{\dagger}\right)$ are the corresponding annihilation (creation) operators with unit vectors $\mathbf{e}_{R}$ and $\mathbf{e}_{L}$, respectively. In the rotating frame, the magnon-photon coupling is given by the interaction Hamiltonian

$$
H_{\mathrm{mp}}=H+H_{\mathrm{ph}}-\mathbf{B}_{p} \cdot \mathbf{S},
$$

where $\mathbf{B}_{p}=\boldsymbol{\nabla} \times \mathbf{A}_{\mathbf{k}}(\mathbf{r}, 0)$ is the photon induced magnetic field interacting through a Zeeman term with the total spin $\mathbf{S}$ of the antiferromagnetic material. $H$ is the spin Hamiltonian [Eq. (1)] and

$$
H_{\mathrm{ph}}=\omega \sum_{\mathbf{k}}\left(c_{\mathbf{k}}^{\dagger} c_{\mathbf{k}}+d_{-\mathbf{k}}^{\dagger} d_{-\mathbf{k}}\right)
$$

is the cavity photon Hamiltonian. For a given $\mathbf{k}$ vector along the $(0,0,1)$ direction, we assume $\mathbf{e}_{R}=-\mathbf{e}_{L}^{*}=\frac{1}{\sqrt{2}}(1,-i, 0)$ and apply the Holstein-Primakoff, Fourier, and Bogoliubov transformations, as mentioned above and described in the
Appendices, to derive the bosonized interaction Hamiltonian

$$
\begin{aligned}
H_{\mathrm{mp} ; \mathbf{k}}= & \omega_{\alpha_{\mathbf{k}}} \alpha_{\mathbf{k}}^{\dagger} \alpha_{\mathbf{k}}+\omega_{\beta_{-\mathbf{k}}} \beta_{-\mathbf{k}}^{\dagger} \beta_{-\mathbf{k}},+\omega\left(c_{\mathbf{k}}^{\dagger} c_{\mathbf{k}}+d_{-\mathbf{k}}^{\dagger} d_{-\mathbf{k}}\right) \\
& +\left(\Delta_{\mathbf{k}} d_{-\mathbf{k}}^{\dagger} \beta_{-\mathbf{k}}+\Delta_{\mathbf{k}}^{*} d_{-\mathbf{k}} \beta_{-\mathbf{k}}^{\dagger}\right) \\
& -\left(\Delta_{\mathbf{k}} c_{\mathbf{k}}^{\dagger} \alpha_{\mathbf{k}}+\Delta_{\mathbf{k}}^{*} c_{\mathbf{k}} \alpha_{\mathbf{k}}^{\dagger}\right)
\end{aligned}
$$

with the resonant magnon-photon interaction. Here $\Delta_{\mathbf{k}}=$ $\lambda_{\mathbf{k}}\left(u_{\mathbf{k}}+v_{\mathbf{k}}^{*}\right)$ for $u_{\mathbf{k}}$ and $v_{\mathbf{k}}$ given by Eq. (A6), $\lambda_{\mathbf{k}}=A_{0} k \sqrt{S}$, and $\mathbf{k}=(0,0, k)$. Note that, apart from the momentum conservation which leads to Eq. (14), for the sake of energy conservation the off-resonant interaction $\left(\Delta_{\mathbf{k}} d_{-\mathbf{k}} \alpha_{\mathbf{k}}+\right.$ $\left.\Delta_{\mathbf{k}}^{*} d_{-\mathbf{k}}^{\dagger} \alpha_{\mathbf{k}}^{\dagger}\right)-\left(\Delta_{\mathbf{k}} c_{\mathbf{k}} \beta_{-\mathbf{k}}+\Delta_{\mathbf{k}}^{*} c_{\mathbf{k}}^{\dagger} \beta_{-\mathbf{k}}^{\dagger}\right)$ is neglected.

Considering the Hamiltonian in Eq. (14), we notice the invariant space spanned by the ordered one-particle Fock states

$$
\begin{aligned}
|1\rangle & =|1000\rangle_{\mathbf{k}}=\alpha_{\mathbf{k}}^{\dagger}|0000\rangle_{\mathbf{k}}, \\
|2\rangle & =|0100\rangle_{\mathbf{k}}=c_{\mathbf{k}}^{\dagger}|0000\rangle_{\mathbf{k}}, \\
\left|1^{\prime}\right\rangle & =|0010\rangle_{\mathbf{k}}=\beta_{-\mathbf{k}}^{\dagger}|0000\rangle_{\mathbf{k}}, \\
\left|2^{\prime}\right\rangle & =|0001\rangle_{\mathbf{k}}=d_{-\mathbf{k}}^{\dagger}|0000\rangle_{\mathbf{k}},
\end{aligned}
$$

with $|0000\rangle_{\mathbf{k}}$ being the joint vacuum state of the magnonphoton system. In this four dimensional invariant subspace, the Hamiltonian takes the following direct sum form:

$$
H_{\mathrm{mp} ; \mathbf{k}}=\left(\begin{array}{cc}
\omega_{\alpha_{\mathbf{k}}} & -\Delta_{\mathbf{k}}^{*} \\
-\Delta_{\mathbf{k}} & \omega
\end{array}\right) \oplus\left(\begin{array}{cc}
\omega_{\beta_{-\mathbf{k}}} & \Delta_{\mathbf{k}}^{*} \\
\Delta_{\mathbf{k}} & \omega
\end{array}\right) .
$$

This implies that we have the following nonzero instantaneous survival and transition probabilities:

$$
\begin{aligned}
|\langle 1|U(t)| 1\rangle|^{2} & =|\langle 2|U(t)| 2\rangle|^{2} \\
& =\cos ^{2}\left[t \pi f_{\mathbf{k}}\right]+\frac{\left(\Delta \omega_{\alpha_{\mathbf{k}}}\right)^{2}}{\left(\Delta \omega_{\alpha_{\mathbf{k}}}\right)^{2}+\left|\Delta_{\mathbf{k}}\right|^{2}} \sin ^{2}\left[t \pi f_{\mathbf{k}}\right], \\
|\langle 2|U(t)| 1\rangle|^{2} & =|\langle 1|U(t)| 2\rangle|^{2} \\
& =\frac{\left|\Delta_{\mathbf{k}}\right|^{2}}{\left(\Delta \omega_{\alpha_{\mathbf{k}}}\right)^{2}+\left|\Delta_{\mathbf{k}}\right|^{2}} \sin ^{2}\left[t \pi f_{\mathbf{k}}\right], \\
\left|\left\langle 1^{\prime}|U(t)| 1^{\prime}\right\rangle\right|^{2} & =\left|\left\langle 2^{\prime}|U(t)| 2^{\prime}\right\rangle\right|^{2} \\
& =\cos ^{2}\left[t \pi f_{\mathbf{k}}^{\prime}\right]+\frac{\left(\Delta \omega_{\beta_{-\mathbf{k}}}\right)^{2}}{\left(\Delta \omega_{\beta_{-\mathbf{k}}}\right)^{2}+\left|\Delta_{\mathbf{k}}\right|^{2}} \sin ^{2}\left[t \pi f_{\mathbf{k}}^{\prime}\right], \\
\left|\left\langle 2^{\prime}|U(t)| 1^{\prime}\right\rangle\right|^{2} & =\left|\left\langle 1^{\prime}|U(t)|^{\prime}\right\rangle\right|^{2} \\
& =\frac{\left|\Delta_{\mathbf{k}}\right|^{2}}{\left(\Delta \omega_{\beta_{-\mathbf{k}}}\right)^{2}+\left|\Delta_{\mathbf{k}}\right|^{2}} \sin ^{2}\left[t \pi f_{\mathbf{k}}^{\prime}\right],
\end{aligned}
$$

where $U(t)=e^{-i t H_{\mathrm{mp}}}$ is the time evolution operator and $\Delta \omega_{\alpha_{\mathbf{k}}}=\frac{\omega_{\alpha_{\mathbf{k}}}-\omega}{2}$ and $\Delta \omega_{\beta_{-\mathbf{k}}}=\frac{\omega_{\beta_{-}-\mathbf{k}}-\omega}{2}$ are the magnon-photon energy differences. Transition frequencies read $\pi f_{\mathbf{k}}=\frac{\pi}{T_{\mathbf{k}}}=$ $\sqrt{\left(\Delta \omega_{\alpha_{\mathbf{k}}}\right)^{2}+\left|\Delta_{\mathbf{k}}\right|^{2}}$ and $\pi f_{\mathbf{k}}^{\prime}=\frac{\pi}{T_{\mathbf{k}}^{\prime}}=\sqrt{\left(\Delta \omega_{\beta_{-\mathbf{k}}}\right)^{2}+\left|\Delta_{\mathbf{k}}\right|^{2}}$, which result explicitly in relations to the EPR function:

$$
\begin{aligned}
\Delta_{0}\left(r_{\mathbf{k}}, \phi_{\mathbf{k}}\right) & =\frac{\left(\pi f_{\mathbf{k}}\right)^{2}-\left(\Delta \omega_{\alpha_{\mathbf{k}}}\right)^{2}}{\lambda_{\mathbf{k}}^{2}} \\
& =\frac{\left(\pi f_{\mathbf{k}}^{\prime}\right)^{2}-\left(\Delta \omega_{\beta_{-\mathbf{k}}}\right)^{2}}{\lambda_{\mathbf{k}}^{2}} .
\end{aligned}
$$


This, in fact, follows from

$$
\begin{aligned}
\frac{\left|\Delta_{\mathbf{k}}\right|^{2}}{\lambda_{\mathbf{k}}^{2}}=\left|u_{\mathbf{k}}+v_{\mathbf{k}}^{*}\right|^{2} & =\cosh 2 r_{\mathbf{k}}+\sinh 2 r_{\mathbf{k}} \cos \phi_{\mathbf{k}} \\
& =\Delta_{0}\left(r_{\mathbf{k}}, \phi_{\mathbf{k}}\right) .
\end{aligned}
$$

Equations (17) and (18) indicate that by tuning the photon frequency $\omega$ to either of the magnon frequencies $\omega_{\alpha_{k}}$ or $\omega_{\beta_{-k}}$, the EPR function and consequently the EPR nonlocality and the degree of magnon-magnon entanglement can be measured through the relevant transition frequency $f_{\mathbf{k}}$ or $f_{\mathbf{k}}^{\prime}$, where the associated magnon-photon transition intensity between $1 \leftrightarrow 2$ or $1^{\prime} \leftrightarrow 2^{\prime}$, i.e., the visibility of magnon-photon interference fringes, is maximal. From Eq. (17) we note that the transition frequencies are the same as the corresponding survival frequencies. Therefore, the measurement of instantaneous survival probability $|\langle x|U(t)| x\rangle|^{2}$ for each magnon or photon, where $|x\rangle$ represents the one-particle Fock states $|1\rangle,|2\rangle,\left|1^{\prime}\right\rangle$, or $\left|2^{\prime}\right\rangle$, also allows for quantification of the EPR function.

A key practical feature is the direct sum form of the Hamiltonian in Eq. (16), which results from energy and momentum conservation mentioned above. This assures that there are no transitions between the two subspaces $\mathcal{M}=\operatorname{span}\{|1\rangle,|2\rangle\}$ and $\mathcal{M}^{\prime}=\operatorname{span}\left\{\left|1^{\prime}\right\rangle,\left|2^{\prime}\right\rangle\right\}$ at any time. This implies that each of the polarized photons independently interacts with its hybridized $\alpha$ or $\beta$ magnon-mode counterpart. This feature together with Eqs. (17) and (18) indicates that, in fact, only a single circularly polarized cavity field is needed to measure the EPR function through magnon-photon transition frequency.

Regarding the actual measurement, a correct interpretation of quantities in Eq. (17) is needed to devise the measurement technique. One may notice that these quantities indeed describe probabilities, as they take their values between 0 and 1 , and also the first two as well as the last two expressions corresponding to the two subspaces $\mathcal{M}$ and $\mathcal{M}^{\prime}$ each add up to 1 . In particular, each of the photon-magnon transition probabilities at a given time is just the probability at which the corresponding polarized single photon is absorbed by the antiferromagnetic material. By this explanation, one can see that the transition probabilities are measurable quantities using polarization sensitive single photon detectors that measure the fluctuation between one and zero photon in the cavity. In other words, the transition probabilities can be viewed as expectation values of the projection operators corresponding to the location of the single photon detectors.

Figure 3 (left panel) shows the measurement scheme associated with the subspace $\mathcal{M}$. In this figure, a quantized circularly polarized photon is depicted to interact with the hybridized magnon mode ( $\alpha$ mode) in a microwave cavity. By a careful control of the magnon and photon frequencies, one can measure the EPR function through the transition frequency $f_{\mathbf{k}}=1 / T_{\mathbf{k}}$ between the magnonic $\alpha$ mode and the interacting photonic mode, and therefore evaluate the degree of bipartite continuous-variable magnon-magnon entanglement. In the right panels of Fig. 3, this is illustrated via the transition probability (lower right panel) between magnon and photon modes, as a function of time. The oscillation period, $T_{\mathrm{k}}$, of the magnon-photon transition probability, which can be measured via a photon counting technique, determines the
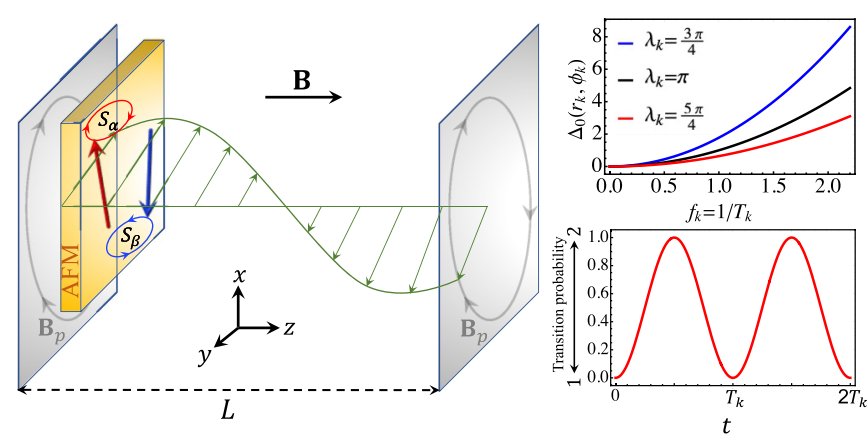

FIG. 3. Left panel: A microwave cavity consisting of two perfect conductor plates located on the $z$ axis at a distance $L$ from each other. The hybridized magnon in an antiferromagnet (schematically shown by the yellow slab denoted by AFM), which combines two ferromagnetic spin sublattices with opposite magnetization along the $z$ direction, is coupled to a quantized circularly polarized cavity photon field (green curve, including arrows). Right panels: The resonant magnon-photon coupling allows for measuring the EPR function $\Delta_{0}\left(r_{\mathbf{k}}, \phi_{\mathbf{k}}\right)$ via the magnon-photon transition frequency $f_{\mathbf{k}}$. The oscillation period $T_{\mathbf{k}}$ of the magnon-photon transition probability determines the transition frequency. An external magnetic field tunes the resonance frequency of the magnon while the photon frequency depends on the ratio between the speed of light and the separation distance $L$.

transition frequency $f_{\mathbf{k}}$. The figure also shows the EPR function as a function of $f_{\mathbf{k}}=1 / T_{\mathbf{k}}$ [explicitly defined in Eq. (18) and below] for selected values of $\lambda_{\mathbf{k}}$ (upper right panel). A similar $\mathcal{M}^{\prime}$-based setup is equally valid.

\section{CONCLUSION}

In conclusion, we have shown that an antiferromagnetic coupling between two ferromagnetic sublattices creates magnon-magnon entanglement in such a way that each energy eigenstate becomes a two-magnon-mode entangled state. The bipartite magnon-mode entanglement is fully characterized by a single entangling parameter, which is clearly related to the EPR function of the ground state. We propose a feasible measurement setup based on light and matter interaction to quantify the EPR function through measurement of the magnon-photon transition frequency. The proposed setup is compatible with current advances in magnonic and photonic technologies.

\section{ACKNOWLEDGMENTS}

The authors acknowledge financial support from the Knut and Alice Wallenberg Foundation through Grant No. 2018.0060. A.D. acknowledges financial support from the Swedish Research Council through Grants No. 2015-04608, No. 2016-05980, and No. 2019-05304. O.E. acknowledges support from the Swedish Research Council, the Swedish Foundation for Strategic Research, the Swedish Energy Agency, European Research Council (synergy grant FASTCORR, Project No. 854843), eSSENCE, and STandUPP. D.T. acknowledges support from the Swedish Research Council through Grant No. 2019-03666. E.S. acknowledges financial support from the Swedish Research Council through 
Grant No. 2017-03832. Some of the computations were performed on resources provided by the Swedish National Infrastructure for Computing at the National Supercomputer Center, Linköping University, the PDC Centre for High Performance Computing at KTH Royal Institute of Technology, and the High Performance Computing Center North at Umeå University.

\section{APPENDIX A: DESCRIPTION OF THE HAMILTONIAN IN TERMS OF BOSONIC OPERATORS}

The magnetic interactions of the system are described by the Hamiltonian in Eqs. (1) and (2), as detailed in the main text. To bosonize the Hamiltonian in terms of collective modes in $\mathbf{k}$ space (see Ref. [35] for a review), we use the HolsteinPrimakoff transformation,

$$
\begin{aligned}
& \text { Sublattice } A:\left\{\begin{array}{l}
S_{i}^{z}=S-a_{i}^{\dagger} a_{i}, \\
S_{i}^{+}=\left(2 S-a_{i}^{\dagger} a_{i}\right)^{\frac{1}{2}} a_{i}, \\
S_{i}^{-}=a_{i}^{\dagger}\left(2 S-a_{i}^{\dagger} a_{i}\right)^{\frac{1}{2}},
\end{array}\right. \\
& \text { Sublattice } B:\left\{\begin{array}{l}
S_{j}^{z}=b_{j}^{\dagger} b_{j}-S, \\
S_{j}^{+}=b_{j}^{\dagger}\left(2 S-b_{j}^{\dagger} b_{j}\right)^{\frac{1}{2}}, \\
S_{j}^{-}=\left(2 S-b_{j}^{\dagger} b_{j}\right)^{\frac{1}{2}} b_{j},
\end{array}\right.
\end{aligned}
$$

and linear approximation at low temperatures $\left(k_{B} T \ll\right.$ $\min \left\{J^{(1)}, J^{(2)}\right\}$ ), where $\left\langle a_{i}^{\dagger} a_{i}\right\rangle \ll S$ and $\left\langle b_{j}^{\dagger} b_{j}\right\rangle \ll S$, followed by Fourier transformation

$$
\begin{aligned}
& a_{i}=\sqrt{\frac{2}{N}} \sum_{\mathbf{k}} e^{-i \mathbf{k} \cdot \mathbf{r}_{i}} a_{\mathbf{k}} \Leftrightarrow a_{\mathbf{k}}=\sqrt{\frac{2}{N}} \sum_{i} e^{i \mathbf{k} \cdot \mathbf{r}_{i}} a_{i}, \\
& b_{j}=\sqrt{\frac{2}{N}} \sum_{\mathbf{k}^{\prime}} e^{-i \mathbf{k}^{\prime} \cdot \mathbf{r}_{j}} b_{\mathbf{k}^{\prime}} \Leftrightarrow b_{\mathbf{k}^{\prime}}=\sqrt{\frac{2}{N}} \sum_{j} e^{i \mathbf{k}^{\prime} \cdot \mathbf{r}_{j}} b_{j}
\end{aligned}
$$

with orthogonality relations

$$
\sum_{i} e^{ \pm i\left(\mathbf{k}-\mathbf{k}^{\prime}\right) \cdot \mathbf{r}_{i}}=\frac{N}{2} \delta_{\mathbf{k} \mathbf{k}^{\prime}}, \quad \sum_{\mathbf{k}} e^{ \pm i \mathbf{k} \cdot\left(\mathbf{r}_{j}-\mathbf{r}_{i}\right)}=\frac{N}{2} \delta_{i j} .
$$

Here, $N$ is the number of sites and

$$
\gamma_{\mathbf{k}}^{(1)}=\frac{1}{z^{(1)}} \sum_{\delta_{1}} e^{i \mathbf{k} \cdot \delta_{1}}, \quad \gamma_{\mathbf{k}}^{(2)}=\frac{1}{z^{(2)}} \sum_{\delta_{2}} e^{i \mathbf{k} \cdot \delta_{2}},
$$

where the sums are carried out over the vectors $\boldsymbol{\delta}_{1}$ connecting a magnetic site to its nearest neighbors and the vectors $\boldsymbol{\delta}_{2}$ connecting a magnetic site to its next-nearest neighbors. $z^{(1)}$ and $z^{(2)}$ are the numbers of nearest and next-nearest neighbors of each site on the lattice, respectively. The bosonized Hamiltonian in $\mathbf{k}$ space reads

$$
\begin{aligned}
H_{\mathbf{k}}= & \omega_{a_{\mathbf{k}}} a_{\mathbf{k}}^{\dagger} a_{\mathbf{k}}+\omega_{b_{-\mathbf{k}}} b_{-\mathbf{k}}^{\dagger} b_{-\mathbf{k}} \\
& +g_{\mathbf{k}} a_{\mathbf{k}} b_{-\mathbf{k}}+g_{\mathbf{k}}^{*} a_{\mathbf{k}}^{\dagger} b_{-\mathbf{k}}^{\dagger},
\end{aligned}
$$

where

$$
\begin{aligned}
\omega_{a_{\mathbf{k}}}= & \epsilon_{\mathbf{k}}-B, \quad \omega_{b_{-\mathbf{k}}}=\epsilon_{\mathbf{k}}+B \\
\epsilon_{\mathbf{k}}= & S\left(z^{(1)} J^{(1)}+2 \mathcal{K}\right. \\
& \left.+z^{(2)}\left\{J^{(2)}-2 \operatorname{Re}\left[\left(J^{(2)}-i D^{(2)}\right) \gamma_{\mathbf{k}}^{(2)}\right]\right\}\right), \\
g_{\mathbf{k}}= & S z^{(1)} \gamma_{\mathbf{k}}^{(1)}\left(J^{(1)}+i D^{(1)}\right) .
\end{aligned}
$$

Here, $a_{\mathbf{k}}^{\dagger}\left(a_{\mathbf{k}}\right)$ and $b_{-\mathbf{k}}^{\dagger}\left(b_{-\mathbf{k}}\right)$ are bosonic creation (annihilation) operators, which mutually commute and define independent families of bosonic operators on the opposite sublattices $A$ and $B$, respectively.

Under SU(1,1) Bogoliubov transformation

$$
\left(\begin{array}{c}
a_{\mathbf{k}} \\
b_{-\mathbf{k}}^{\dagger}
\end{array}\right)=\left(\begin{array}{cc}
u_{\mathbf{k}} & v_{\mathbf{k}} \\
v_{\mathbf{k}}^{*} & u_{\mathbf{k}}^{*}
\end{array}\right)\left(\begin{array}{c}
\alpha_{\mathbf{k}} \\
\beta_{-\mathbf{k}}^{\dagger}
\end{array}\right)
$$

where $u_{\mathbf{k}}=\cosh \left(r_{\mathbf{k}}\right)$ and $v_{\mathbf{k}}=\sinh \left(r_{\mathbf{k}}\right) e^{i \phi_{\mathbf{k}}}$ with

$$
\begin{aligned}
& r_{\mathbf{k}}=\tanh ^{-1}\left[\frac{1-\sqrt{1-\left|\Gamma_{\mathbf{k}}\right|^{2}}}{\left|\Gamma_{\mathbf{k}}\right|}\right] \geqslant 0, \\
& \phi_{\mathbf{k}}=\pi-\arg \left[\Gamma_{\mathbf{k}}\right], \quad \Gamma_{\mathbf{k}}=\frac{2 g_{\mathbf{k}}}{\omega_{a_{\mathbf{k}}}+\omega_{b_{-\mathbf{k}}}}=\frac{g_{\mathbf{k}}}{\epsilon_{\mathbf{k}}},
\end{aligned}
$$

we obtain the following diagonal form of the Hamiltonian:

$$
H_{\mathbf{k}}=\omega_{\alpha_{\mathbf{k}}} \alpha_{\mathbf{k}}^{\dagger} \alpha_{\mathbf{k}}+\omega_{\beta_{-\mathbf{k}}} \beta_{-\mathbf{k}}^{\dagger} \beta_{-\mathbf{k}}
$$

with magnon dispersion relation

$$
\begin{aligned}
\omega_{\alpha_{\mathbf{k}}} & =\tilde{\epsilon}_{\mathbf{k}}-B, \quad \omega_{\beta_{\mathbf{k}}}=\tilde{\epsilon}_{\mathbf{k}}+B, \\
\tilde{\epsilon}_{\mathbf{k}} & =\cosh \left(2 r_{\mathbf{k}}\right) \epsilon_{\mathbf{k}}+\sinh \left(2 r_{\mathbf{k}}\right) \operatorname{Re}\left(g_{\mathbf{k}} e^{i \phi_{\mathbf{k}}}\right)
\end{aligned}
$$

provided $\left|\Gamma_{\mathbf{k}}\right|<1$. The $\mathrm{SU}(1,1)$ condition $\left|u_{\mathbf{k}}\right|^{2}-\left|v_{\mathbf{k}}\right|^{2}=1$ assures that $\alpha_{\mathbf{k}}$ and $\beta_{-\mathbf{k}}$ for all $\mathbf{k}$ also define independent families of bosonic operators.

\section{APPENDIX B: DESCRIPTION OF MAGNON-MAGNON ENTANGLEMENT IN TERMS OF THE ENTANGLEMENT PARAMETER}

From the diagonal expression in Eq. (A8), the ground state of the Hamiltonian $H_{\mathbf{k}}$ in the $(\alpha, \beta)$ mode reads

$$
\left|\psi_{0}\right\rangle=\left|0 ; \alpha_{\mathbf{k}}\right\rangle\left|0 ; \beta_{-\mathbf{k}}\right\rangle
$$

where $\left|0 ; \alpha_{\mathbf{k}}\right\rangle$ and $\left|0 ; \beta_{-\mathbf{k}}\right\rangle$ are vacuum states of $\alpha_{\mathbf{k}}$ and $\beta_{-\mathbf{k}}$, respectively, i.e.,

$$
\alpha_{\mathbf{k}}\left(\left|0 ; \alpha_{\mathbf{k}}\right\rangle\left|0 ; \beta_{-\mathbf{k}}\right\rangle\right)=\beta_{-\mathbf{k}}\left(\left|0 ; \alpha_{\mathbf{k}}\right\rangle\left|0 ; \beta_{-\mathbf{k}}\right\rangle\right)=0 .
$$

By expressing the product vacuum state as a linear combination of $\left|n ; a_{\mathbf{k}}\right\rangle$ and $\left|n ; b_{-\mathbf{k}}\right\rangle$, which are the occupation number bases for the bosonic operators $a_{\mathbf{k}}$ and $b_{-\mathbf{k}}$, respectively, i.e.,

$$
\left|0 ; \alpha_{\mathbf{k}}\right\rangle\left|0 ; \beta_{-\mathbf{k}}\right\rangle=\sum_{n=0}^{\infty} p_{n ; \mathbf{k}}\left|n ; a_{\mathbf{k}}\right\rangle\left|n ; b_{-\mathbf{k}}\right\rangle,
$$

and by inserting it into Eq. (B2), we find

$$
p_{n+1 ; \mathbf{k}}=\frac{v_{\mathbf{k}}}{u_{\mathbf{k}}^{*}} p_{n ; \mathbf{k}}
$$

where $v_{\mathbf{k}}$ and $u_{\mathbf{k}}$ are given by Bogoliubov transformation in Eq. (A6). By solving this recursive equation with normalization constraint $\sum_{n=0}^{\infty}\left|p_{n ; \mathbf{k}}\right|^{2}=1$, the probability amplitudes in the superposed coherent state of Eq. (B3) become

$$
p_{n ; \mathbf{k}}=\frac{e^{i n \phi_{\mathbf{k}}}}{\cosh r_{\mathbf{k}}} \tanh ^{n} r_{\mathbf{k}} .
$$


Therefore, in the $(a, b)$ modes, the ground state magnetic modes can be written as a vector-valued function of $\left(r_{\mathbf{k}}, \phi_{\mathbf{k}}\right)$ :

$$
\left|\psi_{0}\left(r_{\mathbf{k}}, \phi_{\mathbf{k}}\right)\right\rangle=\frac{1}{\cosh r_{\mathbf{k}}} \sum_{n=0}^{\infty} e^{i n \phi_{\mathbf{k}}} \tanh ^{n} r_{\mathbf{k}}\left|n ; a_{\mathbf{k}}\right\rangle\left|n ; b_{-\mathbf{k}}\right\rangle .
$$

Note that we have in fact performed the inverse of the transformation in Eq. (A6) to derive Eq. (B6) from Eq. (B1).

Equations (B1) and (B6) indicate that while the ground state is a product state in $(\alpha, \beta)$ modes, it is an entangled state when expressed in $(a, b)$ modes with the entanglement entropy given by

$$
\begin{aligned}
E\left[\left|\psi_{0}\left(r_{\mathbf{k}}, \phi_{\mathbf{k}}\right)\right\rangle\right]= & {\left[\cosh ^{2}\left(r_{\mathbf{k}}\right) \log \cosh ^{2}\left(r_{\mathbf{k}}\right)\right.} \\
& \left.-\sinh ^{2}\left(r_{\mathbf{k}}\right) \log \sinh ^{2}\left(r_{\mathbf{k}}\right)\right] .
\end{aligned}
$$

A hierarchy of magnon-mode entanglement in antiferromagnets has been studied in Ref. [11]. Note that the entanglement is quantified by the parameter $r_{\mathbf{k}}$, or equivalently $\left|\Gamma_{\mathbf{k}}\right|$. This is indeed true for the complete energy eigenbasis states, which

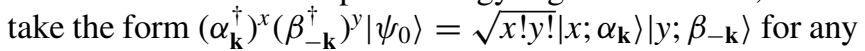
positive integer powers of $x$ and $y$ that, although each of them is disentangled in the $(\alpha, \beta)$ modes, are entangled states in the $(a, b)$ magnon modes with an entanglement entropy that only depends on the parameter $r_{\mathbf{k}}$, or equivalently $\left|\Gamma_{\mathbf{k}}\right|$. To see this, we note that

$$
\begin{aligned}
& \left(\alpha_{\mathbf{k}}^{\dagger}\right)^{x}\left(\beta_{-\mathbf{k}}^{\dagger}\right)^{y}\left|\psi_{0}\right\rangle \equiv \sum_{n=0}^{\infty} p_{n ; \mathbf{k}}^{(x, y)}\left|n+\delta m ; a_{\mathbf{k}}\right\rangle\left|n ; b_{-\mathbf{k}}\right\rangle, x \geqslant y, \\
& \left(\alpha_{\mathbf{k}}^{\dagger}\right)^{x}\left(\beta_{-\mathbf{k}}^{\dagger}\right)^{y}\left|\psi_{0}\right\rangle \equiv \sum_{n=0}^{\infty} p_{n ; \mathbf{k}}^{(x, y)}\left|n ; a_{\mathbf{k}}\right\rangle\left|n+\delta m ; b_{-\mathbf{k}}\right\rangle, x \leqslant y,
\end{aligned}
$$

by induction and $\delta m=|x-y|$. Here, the probability amplitudes are given by

$$
p_{n ; \mathbf{k}}^{(x, y)}=\frac{1}{\sqrt{x ! y !}}\left(\frac{1}{u_{\mathbf{k}}^{*}}\right)^{\delta m}\left(\frac{1}{u_{\mathbf{k}}^{*} v_{\mathbf{k}}}\right)^{m} q_{n ; \mathbf{k}}^{(m, \delta m)} p_{n ; \mathbf{k}},
$$

where $m=\min \{x, y\}$ and $p_{n: \mathbf{k}}$ is the expansion coefficient given in Eq. (B5). $q_{n ; \mathbf{k}}^{(m, \delta m)}$ satisfies the following recursive relations:

$$
\begin{aligned}
q_{n ; \mathbf{k}}^{(m, \delta m>0)}= & \left|u_{\mathbf{k}}\right|^{2} \sqrt{n+\delta m} q_{n ; \mathbf{k}}^{(m, \delta m-1)} \\
& -\left|v_{\mathbf{k}}\right|^{2} \sqrt{n+1} q_{n+1 ; \mathbf{k}}^{(m, \delta m-1)} \\
q_{n ; \mathbf{k}}^{(m>0,0)}= & n\left|u_{\mathbf{k}}\right|^{4} q_{n-1 ; \mathbf{k}}^{(m-1,0)}-(2 n+1)\left|u_{\mathbf{k}} v_{\mathbf{k}}\right|^{2} q_{n ; \mathbf{k}}^{(m-1,0)} \\
& +(n+1)\left|v_{\mathbf{k}}\right|^{4} q_{n+1 ; \mathbf{k}}^{(m-1,0)},
\end{aligned}
$$

with initial value condition $q_{n ; \mathbf{k}}^{(0,0)}=1$ for each $n$.

Since $\left|u_{\mathbf{k}}\right|,\left|v_{\mathbf{k}}\right|$, and the probabilities $\left|p_{n ; \mathbf{k}}\right|^{2}$ are only functions of $r_{\mathbf{k}}$, the probabilities $\left|p_{n ; \mathbf{k}}^{(x, y)}\right|^{2}$ are only functions of $r_{\mathbf{k}}$. This demonstrates that the entanglement entropies of all energy eigenbasis states in the $(a, b)$ modes, i.e.,

$$
E\left[\left(\alpha_{\mathbf{k}}^{\dagger}\right)^{x}\left(\beta_{-\mathbf{k}}^{\dagger}\right)^{y}\left|\psi_{0}\right\rangle\right]=-\sum_{n=0}^{\infty}\left|p_{n ; \mathbf{k}}^{(x, y)}\right|^{2} \log \left|p_{n ; \mathbf{k}}^{(x, y)}\right|^{2},
$$

are only functions of the single squeezing parameter $r_{\mathbf{k}}$. For this reason we identify $r_{\mathbf{k}}$, or equivalently $\left|\Gamma_{\mathbf{k}}\right|$, as the magnonmagnon entanglement parameter of the system.

Note that $H_{1}=0$ corresponds to $r_{\mathbf{k}}=0$ and trivial Bogoliubov transformation in Eq. (A6). In this case the $(a, b)$ and $(\alpha, \beta)$ modes coincide and therefore both Hamiltonians in Eqs. (3) and (5) describe two separate noninteracting ferromagnets with opposite magnetizations. Moreover, the full energy spectra are given by product states $\left|n ; a_{\mathbf{k}}\right\rangle\left|m ; b_{-\mathbf{k}}\right\rangle$, which indicate $n$ and $m$ ferromagnetic magnons in the bosonic modes of $a \equiv \alpha$ and $b \equiv \beta$, respectively. However, for $H_{1} \neq$ 0 , the nontrivial transformation in Eq. (A6) combines the two oppositely magnetized ferromagnetic magnon modes of $a$ and $b$, which represent two ferromagnetic sublattices, into antiferromagnetic magnon modes $(\alpha, \beta)$. Thus, in the presence of antiferromagnetic interaction $H_{1}$, the trivial entanglement entropy between $\alpha$ and $\beta$ modes means no entanglement between antiferromagnetic magnons in the system while nonzero entanglement entropy in $(a, b)$ modes indicates entanglement between the two ferromagnetic magnons or the two ferromagnetic sublattices.
[1] D. D. Awschalom, C. H. R. Du, R. He, F. J. Heremans, A. Hoffmann, J. T. Hou, H. Kurebayashi, Y. Li, L. Liu, V. Novosad, J. Sklenar, S. E. Sullivan, D. Sun, H. Tang, V. Tiberkevich, C. Trevillian, A. W. Tsen, L. R. Weiss, W. Zhang, X. Zhang, L. Zhao, and C. W. Zollitsch, Quantum engineering with hybrid magnonics systems and materials, IEEE Trans. Quantum Eng. 2, 5500836 (2021).

[2] Y. Li, W. Zhang, V. Tyberkevych, W.-K. Kwok, A. Hoffmann, and V. Novosad, Hybrid magnonics: Physics, circuits, and applications for coherent information processing, J. Appl. Phys. 128, 130902 (2020).

[3] D. Lachance-Quirion, Y. Tabuchi, A. Gloppe, K. Usami, and Y. Nakamura, Hybrid quantum systems based on magnonics, Appl. Phys. Express 12, 070101 (2019).
[4] A. A. Clerk, K. W. Lehnert, P. Bertet, J. R. Petta, and Y. Nakamura, Hybrid quantum systems with circuit quantum electrodynamics, Nat. Phys. 16, 257 (2020).

[5] J. Li, S.-Y. Zhu and G. S. Agarwal, Magnon-Photon-Phonon Entanglement in Cavity Magnomechanics, Phys. Rev. Lett. 121, 203601 (2018).

[6] J. Li and S.-Y. Zhu, Entangling two magnon modes via magnetostrictive interaction, New J. Phys. 21, 085001 (2019).

[7] Z. Zhang, M. O. Scully, and G. S. Agarwal, Quantum entanglement between two magnon modes via Kerr nonlinearity driven far from equilibrium, Phys. Rev. Research 1, 023021 (2019).

[8] D. Bossini, S. Dal Conte, G. Cerullo, O. Gomonay, R. V. Pisarev, M. Borovsak, D. Mihailovic, J. Sinova, J. H. Mentink, 
Th. Rasing, and A. V. Kimel, Laser-driven quantum magnonics and terahertz dynamics of the order parameter in antiferromagnets, Phys. Rev. B 100, 024428 (2019).

[9] H. Y. Yuan, S. Zheng, Z. Ficek, Q. Y. He, and M.-H. Yung, Enhancement of magnon-magnon entanglement inside a cavity, Phys. Rev. B 101, 014419 (2020).

[10] H. Y. Yuan, P. Yan, S. Zheng, Q. Y. He, K. Xia, and M.-H. Yung, Steady Bell State Generation via Magnon-Photon Coupling, Phys. Rev. Lett. 124, 053602 (2020).

[11] V. Azimi-Mousolou, A. Bagrov, A. Bergman, A. Delin, O. Eriksson, Y. Liu, M. Pereiro, D. Thonig, and E. Sjüqvist, Hierarchy of magnon mode entanglement in antiferromagnets, Phys. Rev. B 102, 224418 (2020).

[12] B. Lenk, H. Ulrichs, F. Garbs, and M. Münzenberg, The building blocks of magnonics, Phys. Rep. 507, 107 (2011).

[13] B. D. Cullity and C. D. Graham, Introduction to Magnetic Materials (Wiley Online Library, 2009).

[14] J. Stöhr and H. C. Siegmann, Magnetism: From Fundamentals to Nanoscale Dynamics (Springer-Verlag, Berlin, 2006).

[15] I. V. Solovyev, Electronic structure and stability of the ferrimagnetic ordering in double perovskites, Phys. Rev. B 65, 144446 (2002).

[16] Y. Tokura and N. Nagaosa, Orbital Physics in Transition-Metal Oxides, Science 288, 462 (2000).

[17] A. Fert, Nobel Lecture: Origin, development, and future of spintronics, Rev. Mod. Phys. 80, 1517 (2008).

[18] G. Giedke, M. M. Wolf, O. Krüger, R. F. Werner, and J. I. Cirac, Entanglement of Formation for Symmetric Gaussian States, Phys. Rev. Lett. 91, 107901 (2003).

[19] M. Fadel, L. Ares, A. Luis, and Q. He, Number-phase entanglement and Einstein-Podolsky-Rosen steering, Phys. Rev. A 101, 052117 (2020).

[20] Y. E. Roginska and Y. N. Venevtse, New magnetic ferroelectrics, Sov. Phys. Solid State USSR 7, 320 (1965).

[21] G. Matsumoto, Study of $\left(\mathrm{La}_{1-x} \mathrm{Ca}_{x}\right) \mathrm{MnO}_{3}$. I. Magnetic Structure of $\mathrm{LaMnO}_{3}$, J Phys. Soc. Jpn. 29, 606 (1970).

[22] C. Gross, H. Strobel, E. Nicklas, T. Zibold, N. Bar-Gill, G. Kurizki, and M. K. Oberthaler, Atomic homodyne detection of continuous-variable entangled twin-atom states, Nature (London) 480, 219 (2011).

[23] S. Armstrong, M. Wang, R. Y. Teh, Q. Gong, Q. He, J. Janousek, H.-A. Bachor, M. D. Reid, and P. K. Lam, Multipartite Einstein-
Podolsky-Rosen steering and genuine tripartite entanglement with optical networks, Nat. Phys. 11, 167 (2015).

[24] J. Peise, I. Kruse, K. Lange, B. Lücke, L. Pezzè, J. Arlt, W. Ertmer, K. Hammerer, L. Santos, A. Smerzi, and C. Klempt, Satisfying the Einstein-Podolsky-Rosen criterion with massive particles, Nat. Commun. 6, 8984 (2015).

[25] J.-C. Lee, K.-K. Park, T.-M. Zhao, and Y.-H. Kim, EinsteinPodolsky-Rosen Entanglement of Narrow-Band Photons from Cold Atoms, Phys. Rev. Lett. 117, 250501 (2016).

[26] P. Kunkel, M. Prüfer, H. Strobel, D. Linnemann, A. Frölian, T. Gasenzer, M. Gärttner, and M. K. Oberthaler, Spatially distributed multipartite entanglement enables EPR steering of atomic clouds, Science 360, 413 (2018).

[27] M. Fadel, T. Zibold, B. Décamps, and P. Treutlein, Spatial entanglement patterns and Einstein-Podolsky-Rosen steering in Bose-Einstein condensates, Science 360, 409 (2018).

[28] J. Li, Y. Liu, N. Huo, L. Cui, S. Feng, X. Li, and Z. Y. Ou, Measuring continuous-variable quantum entanglement with parametric-amplifier-assisted homodyne detection, Phys. Rev. A 101, 053801 (2020).

[29] K. N. Shrivastava, Magnon-photon interaction in antiferromagnets, Phys. Rev. B 19, 1598 (1979).

[30] H. Huebl, C. W. Zollitsch, J. Lotze, F. Hocke, M. Greifenstein, A. Marx, R. Gross, and S. T. B. Goennenwein, High Cooperativity in Coupled Microwave Resonator Ferrimagnetic Insulator Hybrids, Phys. Rev. Lett. 111, 127003 (2013).

[31] Y. Tabuchi, S. Ishino, T. Ishikawa, R. Yamazaki, K. Usami, and Y. Nakamura, Hybridizing Ferromagnetic Magnons and Microwave Photons in the Quantum Limit, Phys. Rev. Lett. 113, 083603 (2014).

[32] H. Y. Yuan and X. R. Wang, Magnon-photon coupling in antiferromagnets, Appl. Phys. Lett. 110, 082403 (2017).

[33] Y. Xiao, X. H. Yan, Y. Zhang, V. L. Grigoryan, C. M. Hu, H. Guo, and K. Xia, Magnon dark mode of an antiferromagnetic insulator in a microwave cavity, Phys. Rev. B 99, 094407 (2019).

[34] Ø. Johansen and A. Brataas, Nonlocal Coupling between Antiferromagnets and Ferromagnets in Cavities, Phys. Rev. Lett. 121, 087204 (2018).

[35] S. M. Rezende, A. Azevedo, and R. L. Rodrigues-Suarez, Introduction to antiferromagnetic magnons, J. Appl. Phys. 126, 151101 (2019). 